\title{
The in vitro effects of artificial and natural sweeteners on the immune system using whole blood culture assays
}

\author{
F. Rahiman and E. J. Pool
}

\begin{abstract}
This article investigates the effects of commercially available artificial (aspartame, saccharin, sucralose) and natural sweeteners (brown sugar, white sugar, molasses) on the immune system. Human whole blood cultures were incubated with various sweeteners and stimulated in vitro with either phytohemagglutinin or endotoxin. Harvested supernatants were screened for cytotoxicity and cytokine release. Results showed that none of the artificial or natural sweeteners proved to be cytotoxic, indicating that no cell death was induced in vitro. The natural sweetener, sugar cane molasses ( $10 \mathrm{ug}=\mathrm{mL})$, enhanced levels of the inflammatory biomarker IL- 6 while all artificial sweeteners ( $10 \mathrm{ug}=\mathrm{mL}$ ) revealed a suppressive effect on IL-6 secretion $(\mathrm{P}<\mathrm{0.001})$. Exposure of blood cells to sucralosecontaining sweeteners under stimulatory conditions reduced levels of the biomarker of humoral immunity, Interleukin-10 $(\mathrm{P}<\mathrm{0.001})$. The cumulative suppression of Interleukin-6 and Interleukin-10 levels induced by sucralose may contribute to the inability in mounting an effective humoral response when posed with an exogenous threat.
\end{abstract}

\section{Introduction}

The desire for sweet tasting food is inherent and characteristic of the human population. Prehistoric man satisfied his need for sweet taste through the intake of certain fruits and vegetables. Years later, through the advancement in food technology, refined sugar was made accessible to people at low cost. This sparked an increase in the use of sugar worldwide. ${ }^{[1]}$

Today, there is an extensive choice of sweeteners available to the consumer. These sweeteners can be categorised into either nutritive (natural sweeteners) or non-nutritive sweeteners (artificially manufactured sweeteners). Sugar cane molasses is an example of a popular, natural sweetener rich in sucrose and is the by-product of the sugar refinement process. ${ }^{\text {[2] }}$ Dating back to the 19th century, molasses has been used widely in livestock and poultry feeds. ${ }^{[3]}$ Today, molasses is increasingly being used as a flavor enhancer, has been substituted as a sweetener, and used as a preservative in jams and jellies. [4] Anecdotal reports also suggest that molasses may be used as a supplement in the human diet to improve conditions such as anaemia, colds, coughs, earaches, 
arthritis, ulcers, hair damage, eczema, high blood pressure, dermatitis, constipation, varicose veins, nerve damage, and bladder problems. ${ }^{[4-6]}$

Although molasses has been associated with various health benefits, there are also reports that suggest the inclusion of molasses in the diet of livestock may induce certain metabolic diseases. Such diseases include molasses toxicity, urea toxicity, and bloat which may occur as a result of molasses being used as a supplement (vehicle for urea) or as the basis of livestock feed. ${ }^{[7]}$ Molasses toxicity is defined as a condition affecting cattle or sheep fed high molasses diets with limited forage. ${ }^{[8]}$ Effected animals suffer from symptoms similar to that of cerebro-ortical necrosis or polioencephalomalacia. Bloat is a condition characterised by the retention of gas in the rumen and occurs in most animal feeding systems. However, this disease appears to be recurrent in diets consisting of carbohydrates supplied by unrefined sugar or maize grain that has little or no fiber, yet is easily digestible. ${ }^{[7]}$

Sugar or sucrose consumption has been associated with dental caries, obesity, and chronic diseases linked to obesity such as cancer, diabetes, and heart disease. As a result, this created a demand for the development of alternate sweeteners, which offer a low-calorie intake. ${ }^{[1]}$ The manufacture of artificial sweeteners by the food industry, promised consumers the sweetness of sugar without the calories. ${ }^{[9]}$ Diabetic patients and individuals wanting to control their weight have access to these products that allow them the pleasure of a sweettasting treat without adverse health effects. ${ }^{[1]}$ These alternate sweeteners are used extensively as additives in food, beverages, confectionary, and in the pharmaceutical sector. Common types of artificial sweeteners include aspartame, acesulfame-K, cyclamate, neotame, sucralose, and saccharin. ${ }^{[10]}$ Although artificial sweeteners have been welcomed into the food industry for its beneficial uses, some studies have found correlations between their use and illnesses such as cancers, hepatotoxicity, headaches, allergies, seizures, diarrhoea, and low birth weight. [9]

Commercially available saccharin is almost 300-500 times sweeter than sucrose. ${ }^{[11]}$ This artificial sweetener is probably one of the most scrupulously researched sweeteners since its discovery, with much of the focus pertaining to its potential role in bladder cancer. $^{[2]}$ There have been approximately 20 experimental groups that have independently investigated the effect of elevated doses of saccharin in one-generation rats. Results from these studies show that the majority of these groups could not positively associate saccharin-fed rats with the development of neoplasias. Although the positive control group did reflect an increased incidence of bladder cancer, this result was questionable since rats that were used in the studies were regularly infected with the bladder parasite Trichosomoides crassicanda. In two-generation rat studies, results show almost 
consistently that rats fed on saccharin diets and born of saccharin-fed parents had an increased risk of developing bladder cancer. ${ }^{[12]}$

Aspartame first came into existence in 1965 and has been packaged under labels such as Nutra sweet ${ }^{\mathrm{TM}}$, Canderel ${ }^{\mathrm{TM}}$, and Equal ${ }^{\mathrm{TM}} \cdot{ }^{[13,14]}$ In the body, aspartame is hydrolyzed into its components, namely, phenylalanine, aspartic acid, and methanol. Research has focused on the plasma levels of these products within the human body. These studies have revealed both potentially normal conditions of use and adverse effects arising from the consumption of aspartame. ${ }^{[15]}$ In 1996, Olney and colleagues published a contentious paper suggesting that a link between the surge in brain tumors since 1980 and the introduction of aspartame into the food industry existed. They further supported their hypothesis by referring to an FDA experiment in which 12 out of 320 Sprague-Dawley rats developed malignant brain tumors following longterm, aspartame ingestion. This hypothesis raised much concern amongst the general public but was also profoundly criticized by many scientists. ${ }^{[12,16]}$

Sucralose has been branded under the name Splenda ${ }^{\mathrm{TM}}$ and has a wide variety of uses in the food industry. ${ }^{[9]}$ The production of sucralose involves the replacement of three chlorine atoms for three hydroxyl groups in sucrose. The safety concern of this compound arises because of the existence of three chlorine atoms, which make it an organochloride. Organochlorides such as pesticides and dioxins have been largely reported as being carcinogenic. Hence, sucralose has also been subjected to toxicity screening. ${ }^{[10]}$

Since their existence, the usage of natural and artificial sweeteners has been quite controversial. Their implication in various diseases has raised much questionability with respect to their safe use. ${ }^{[9]}$ Therefore, it is necessary that these sweeteners be subjected to toxicity screening to determine their effects on human health.

This article aims to determine the potential modulatory effects of both natural and artificial sweeteners on the immune system. Human whole blood cultures (hWBC) were used as a model system to determine the impact of sweeteners on immune function. Lactate dehydrogenase release was used as a biomarker for cytotoxicity. Interleukin-6 (IL-6) was used as a biomarker of the inflammatory response, Interleukin-10 (IL-10) as a biomarker of humoral immunity, and Interferon-gamma (IFNg) as a biomarker of cell-mediated immunity.

The immune system is an intricate and interactive network that comprises of various components. This system primarily functions in defense against foreign or antigenic particles entering the body as well as protecting the integrity of the host against attack and disease triggered by pathogenic organisms. ${ }^{[17]}$ The human immune response consists of two branches, 
which include innate and acquired immunity. Phagocytic cells and cellular components such as interferons make up the innate immune response, which acts as the first line of defense against pathogens. ${ }^{[18,19]}$ The acquired immune response consists of two branches, namely cellmediated and humoral immunity. Both $\mathrm{B}$ and $\mathrm{T}$ lymphocytes function in recognizing and destroying invading pathogens and are the main cells involved in this form of immunity. The acquired immune response requires prior sensitization to an antigen whereas the innate immune response is not dependent on exposure to an antigen. ${ }^{[18]}$

Research in toxicology has shown that the immune system is a target for various chemicals and that a toxicant has the ability to affect a constituent of the immune system at any level. ${ }^{[20,21]}$ Immunotoxicology can be defined as 'the study of adverse effects of foreign substances also referred to as xenobiotics on the immune system, which results in harmful alterations in the host responses and ultimately leads to the increased risk to infectious disease. ${ }^{[20]}$ A chemical insult to the immune system encompasses a variety of effects that include enhancement or suppression of the immune response. ${ }^{[22]}$ A decreased immune response may be associated with an increase in the incidence, time-span, severity, or result of an attack by an invading pathogen. An enhanced immune response may be either beneficial or detrimental to the host since it may further increase the immune response to a disease or neutralise the effects of autoimmunity or hypersensitivity reactions. ${ }^{[17]}$ Industralized countries are experiencing a significant increase in diseases that can be associated with a malfunction in the immune system. ${ }^{[22]}$ Thus, the immunotoxicity of drugs and various compounds, particularly those that we are exposed to regularly, are progressively being identified as potential hazards.

\section{Experimental methods}

\section{Principle of the Procedure}

In this article, human blood was treated with the B-cell mitogen, lipopolysaccharide (LPS), and the T cell mitogen, phytohemagglutinin (PHA). LPS stimulates an inflammatory response and initiates the release of IL-6 from monocytes and B lymphocytes. ${ }^{[24]}$ PHA was used to stimulate the cell mediated and humoral immune pathways, initiating the release of IFNg and IL-10 from T lymphocytes. Cytokine production was measured inthe supernatants, following incubation with or without the test compound. Cytokines were quantified using Enzyme-Linked Immunosorbent Assays (ELISAs).

\section{Whole Blood CytokineAssays}

Blood was obtained from healthy human male donors and was stored at ambient room temperature. Consent was obtained from all participants and all procedures were conducted in accordance with guidelines of the ethics 
committee at the University of the Western Cape. Blood samples were collected in $10 \mathrm{~mL}$ citrate-containing vacuum tubes by venipuncture and used within $8 \mathrm{hr}$ of collection. All procedures were performed under sterile conditions. Whole blood was diluted 1:10 with RPMI-1640 (Sigma-Aldrich Inc., St. Louis, MO, USA) before being stimulated with a mitogen or remaining unstimulated.

Natural (sugar cane molasses, white sugar, and brown sugar) and artificial

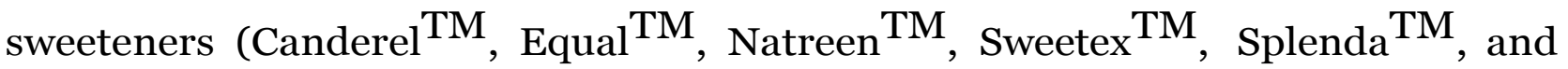
Swheet ${ }^{\mathrm{TM}}$ ) that are commercially available were used for all experiments. The artificial sweeteners used, may be classified into their relevant groups based on their principal ingredient. The brands Canderel ${ }^{\mathrm{TM}}$ and $\mathrm{Equal}^{\mathrm{TM}}$ consist of aspartame, Natreen ${ }^{\mathrm{TM}}$ and Sweetex $^{\mathrm{TM}}$ are composed of saccharin and Splenda $^{\mathrm{TM}}$ and Swheet ${ }^{\mathrm{TM}}$

\section{The Effects of Natural and Artificial Sweeteners on Stimulated and UnstimulatedhWBC}

Diluted whole blood remained unstimulated or stimulated without mitogens (to give a final concentration of $10 \mathrm{ng}=\mathrm{mL}$ LPS and $16 \mathrm{ug}=\mathrm{mL}$ PHA). Both commercially available natural (molasses, white sugar, and brown sugar) and artificial sweeteners (Canderel ${ }^{\mathrm{TM}}$, Equal ${ }^{\mathrm{TM}}$, Natreen ${ }^{\mathrm{TM}}$, Sweetex ${ }^{\mathrm{TM}}$, Splenda ${ }^{\mathrm{TM}}$, and Swheet $^{\mathrm{TM}}$ ) were diluted in distilled water and used at a concentration of $10 \mathrm{ug}=\mathrm{mL}$. LPS-stimulated and unstimulated blood (500 $\mathrm{mL}=$ well) were added to 48 -well culture plates and incubated at $37^{\circ} \mathrm{C}$ in the presence or absence of sweeteners $(5 \mathrm{~mL}=$ well) for $18 \mathrm{hr}$. PHA stimulated and unstimulated blood (500 $\mathrm{mL}=$ well) were added to 48 -well culture plates and incubated at $37^{\circ} \mathrm{C}$ in the presence or absence of sweeteners $(5 \mathrm{~mL}=$ well) for 48 hr. Control wells consisted of stimulated or unstimulated cells cultured in medium alone. All samples were plated in triplicate. At the end of the incubation period, the culture supernatants harvested from the LPS stimulated cultures were assayed for IL-6 and lactate dehydrogenase activity (LDH), while the PHA stimulated supernatants were assayed for IFNg and IL-10 synthesis.

\section{Cytotoxicity}

A commercially available chromogenic, lactate dehydrogenase assay kit was used to analyze all samples (Roche Diagnostics, Indianapolis, IN, USA). The assay was performed in accordance with the manufacturer's guidelines.

\section{Cytokine Analysis}

The concentrations of cytokine biomarkers, i.e., IL-6, IL-10, and IFNg were all determined using commercially available ELISA kits (e-Bioscience, San Diego, CA, USA). All cytokine assays were conducted according to instructions provided. Assays were performed using 96-well plates (Nunc ${ }^{1}$, Serving Life Science, Vernon Hills, IL, USA) and cytokine levels were expressed as $\mathrm{pg}=\mathrm{mL}$. 


\section{Statistical Analysis}

All data was statistically analysed using one-way ANOVA using SigmaStat software (Systat Software Inc., Chicago, IL, USA). The Dunnett's test was specifically used to determine the differences between the sample and the control groups. All cytokine assays were conducted in triplicate to avoid statistical errors. Results were expressed in terms of the mean \pm standard error of the mean (SEM).

\section{Results and discussion}

\section{The Effects of Artificial and Natural Sweeteners on the Immune System Cytotoxicity of Natural and Artificial Sweeteners}

A standard curve was generated to determine the percentage toxicity of all samples. Results showed that percentage toxicity obtained for both stimulated and unstimulated cultures incubated with the various sweeteners, were similar to that of the distilled water control. Hence, none of the natural or artificial sweeteners proved to be cytotoxic (data not shown).

\section{The Effects of Natural and Artificial Sweeteners on Endotoxin-Stimulated and Unstimulated hWBC}

IL-6 is a pleiotrophic cytokine and plays an important role in stimulating B cells to synthesise antibodies. IL-6 is also a sensitive biomarker used to investigate inflammatory activity. ${ }^{[25]}$ A significant effect on IL-6 concentration was observed when comparing individual artificial sweeteners and the natural sweetener, sugar cane molasses to the distilled water control under stimulated conditions $(\mathrm{P}<0.001)$ (Figure 1$)$. In the presence of LPS, all artificial sweeteners suppressed the secretion of IL-6, while sugar cane molasses enhanced the secretion of IL-6 $(\mathrm{P}<0.001)$ in vitro. This result may be interpreted as the inability of artificial sweeteners to produce an effective inflammatory defense when challenged with the bacterial pathogen endotoxin. On the contrary, sugar cane molasses increased inflammatory activity and produces an efficient immune response when posed with a threat. Normally, infection or injury caused to the body initiates the synthesis of pro-inflammatory cytokines and the elevated levels of these cytokines are essential for recovery. ${ }^{[26]}$ The increased inflammatory response produced by molasses under unstimulated conditions may therefore initiate a competent immune defense mounted against invading pathogens on the healthy immune system. However, this form of "hyperstimulation" may also be associated with hypersensitivity reactions, which are known to adversely affect the health of an individual. The ability of molasses to enhance inflammatory activity in vitro is supported by earlier data. ${ }^{[27]}$ As previously stated, the inflammatory potential of molasses may prove to be therapeutic when administered to patients suffering from immunosuppressive illnesses such as cancer and AIDS. ${ }^{[27]}$

In the absence of the stimulus LPS, all artificial sweeteners including the natural sweeteners, brown and white sugar showed no effect on IL-6 synthesis when compared to the distilled water control $(\mathrm{P}>0.001)$. However, IL-6 levels of 
unstimulated cultures exposed to sugar cane molasses exceeded that of the control $(\mathrm{P}<0.001)$.

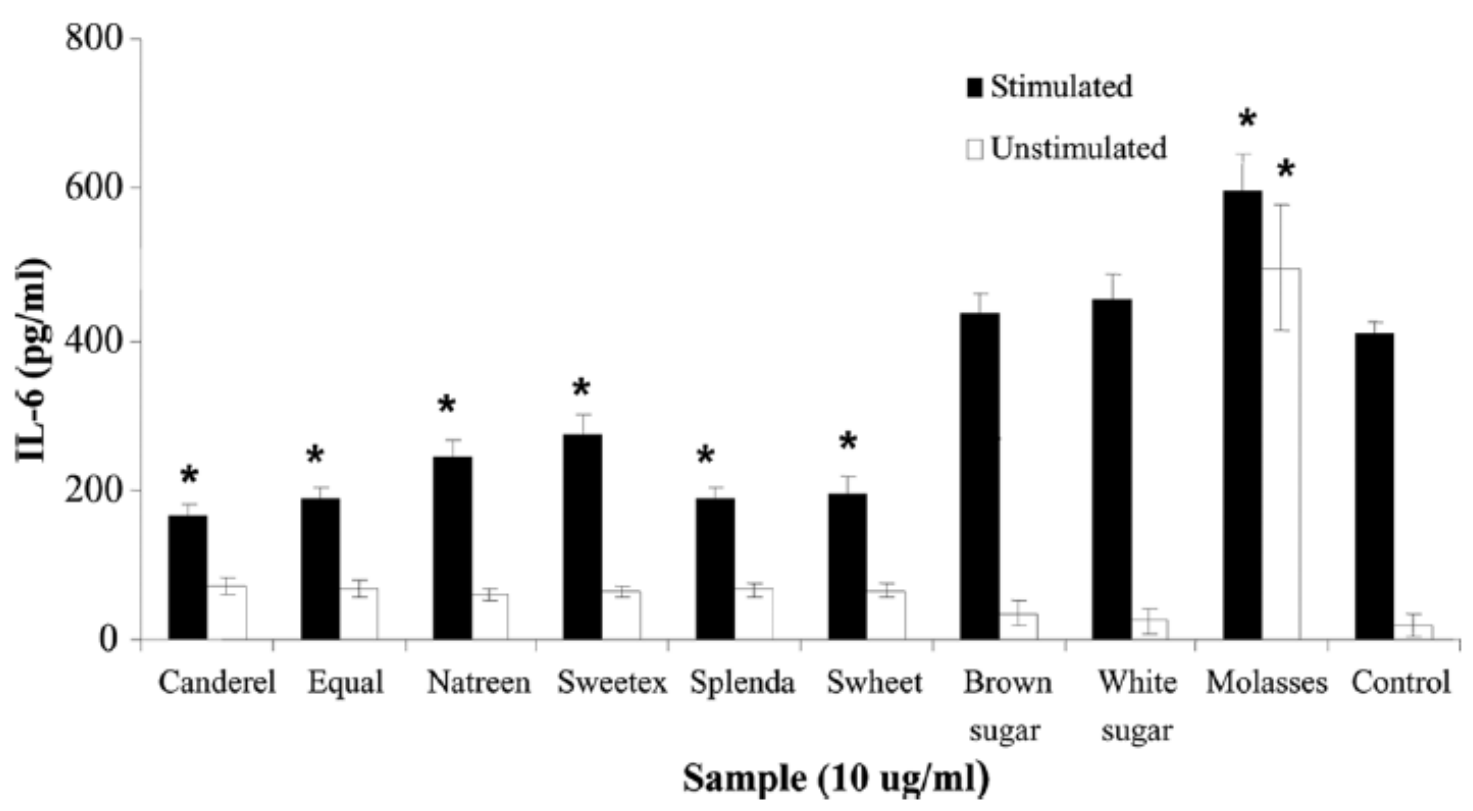

FIGURE 1 The inflammatory activity of artificial and natural sweeteners in LPS stimulated and unstimulated whole blood cultures. Data is expressed as the mean \pm standard error of the mean of three replicates $(n=21)$. An asterisk $(*)$ indicates statistical difference to the distilled water control $(\mathrm{P}<0.001)$. Stimulated $=\mathbf{\square} ;$ unstimulated $=\square$.

The Effects of Natural and Artificial Sweeteners on PHA-Stimulated and Unstimulated hWBC

Certain infections can cause chronic or persistent inflammation that ultimately ends in pathological effects such as cancer, cardiovascular disease, age-related chronic diseases and Type 2 diabetes. ${ }^{[26]}$ The level of cytokine synthesis and the balance between inflammatory cytokines vs. antiinflammatory cytokines determines the time-span as well as the end-result of the immune response. ${ }^{[28]}$

Results showed that the artificial sweetener, sucralose branded under the names of Splenda ${ }^{\mathrm{TM}}$ and Swheet $^{\mathrm{TM}}$ significantly reduced the levels of the anti-inflammatory cytokine, IL-10 under stimulated conditions $(\mathrm{P}<$ o.001) (Figure 2). The decrease of IL-10 levels by sucralose suggests a diminished humoral immune response against extracellular pathogens such as bacteria, fungi and yeast. ${ }^{[29]}$ The cumulative effect of reduced IL6 and IL-10 synthesis induced by sucralose, further suggests the suppression of antibody synthesis against infective extracellular pathogens.

The comparison of all artificial and natural sweeteners to the distilled water control under unstimulated conditions showed no significant effect on IL-6 or IL-1O synthesis (Figures 1 and 2). The Th1 cytokine, IFNg, enhances the 
activation of macrophages, antibody-dependent cellular cytotoxicity as well as the complement pathway. This form of immune protection functions in defense against intracellular pathogens and any disruption caused to these cells may lead to tissue damage in the host. ${ }^{[30]}$ The comparison of all sweeteners to the control cultures under both stimulated and unstimulated conditions showed that none of the sweeteners had an effect on IFNg synthesis ( $P$ > 0.05) (Figure 3). Hence, none of the sweeteners had an impact on cellmediated immunity.

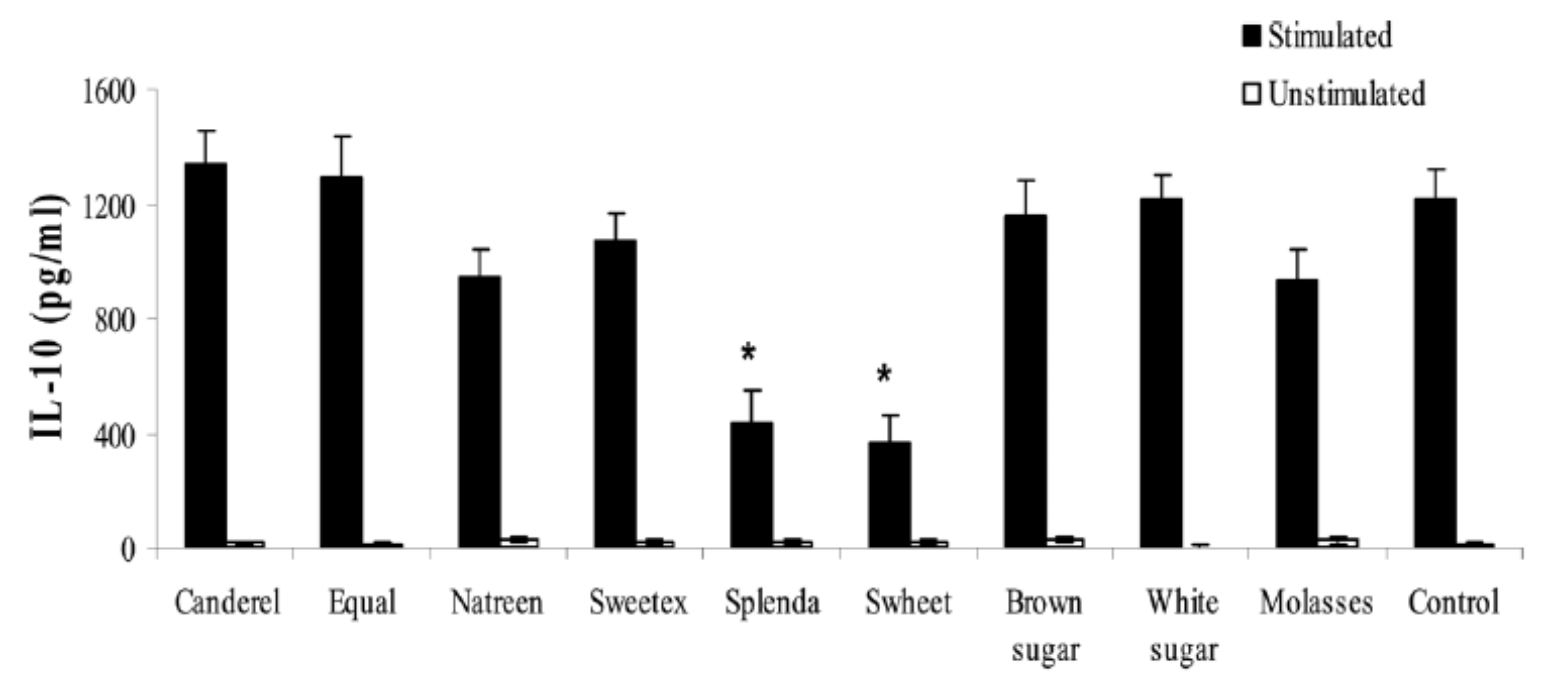

Sample (10 ug/ml)

FIGURE 2 IL-10 synthesis by PHA stimulated and unstimulated WBCs incubated with artificial or natural sweeteners. Data is expressed as the mean \pm standard error of the mean of three replicates ( $\mathrm{n} 1 / 4$ 21). An asterisk (*) indicates the statistical difference to the distilled water control $(\mathrm{P}<0.001)$. Stimulated $=\mathbf{a} ;$ unstimulated $=\square$. 


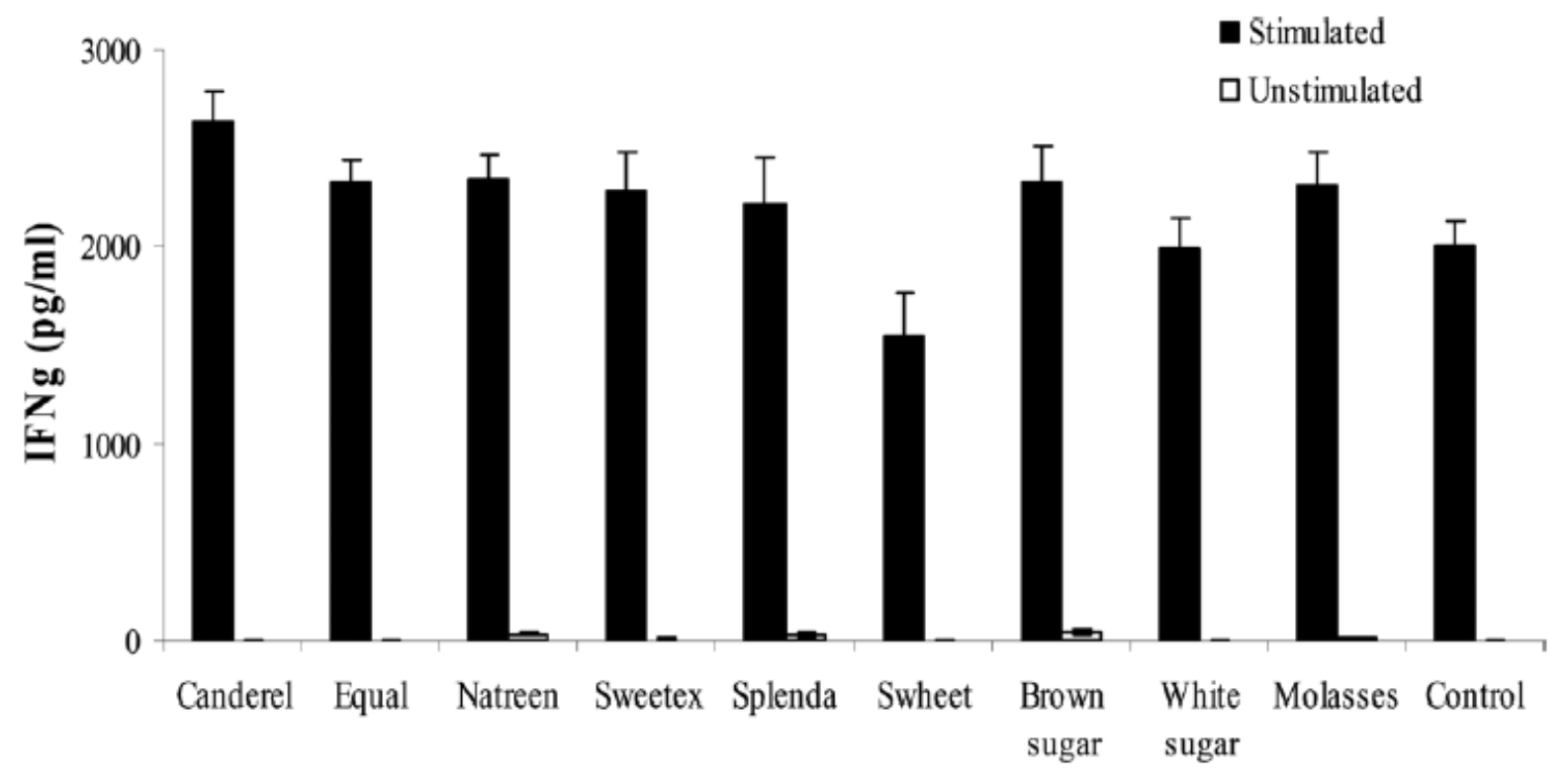

Sample (10 ug/ml)

FIGURE 3 IFNg synthesis by PHA stimulated and unstimulated WBCs incubated with artificial or natural sweeteners. Data is expressed as the mean \pm standard error of the mean of three replicates $(n=21)$. An asterisk $(*)$ indicates the statistical difference to the distilled water control $(P>0.05)$. Stimulated $=\mathbf{m}$; unstimulated $=\square$.

\section{Conclusion}

The current article shows that both artificial and natural sweeteners are not cytotoxic, however they do have an impact on certain cellular pathways. All artificial sweeteners containing aspartame, saccharin and sucralose appear to inhibit inflammatory activity in vitro. Sucralose-containing sweeteners may potentially reduce humoral immunity thus increasing susceptibility of host defense against extracellular pathogens. On the contrary, the inflammatory potential of the nutritive sweetener, sugar cane molasses may be favorable in defense against infective pathogens. Although certain sweeteners have displayed immunomodulatory effects in vitro, further studies on artificial and natural sweeteners are required to determine if these sweeteners display similar effects in vivo.

\section{Acknowledgments}

We are grateful for the financial support received by the National Research Foundation of South Africa, the DAAD (Deutscher Akademishcer Austausch Dienst) foundation in Germany, and the Mellon scholarship by the University of the Western Cape. 


\section{References}

1. Sardesai, V. M.; Waldshan, T. H. Natural and Synthetic Intense Sweeteners. $J$. Nutrit. Biochem. 1991, 2, 236-244.

2. Greenly, L. W. A Doctor's Guide to Sweeteners. J. Chiropr. Med. 2003, 2, 80-86.

3. Curtin, L. V. Molasses-General Considerations. In Molasses in Animal Nutrition; Natl. Feed Ingredients Association: Des Moines, IA, 1983; pp. 1-11.

4. Reyed, R. M.; El-Diwany, A. Molasses as Bifidus Promoter on Bifidobacteria and Lactic Acid Bacteria Growing in Skim Milk. Internet J. Microbiol. [Online] 2008, 5(1). http:// www.ispub.com/journal/the internet_journal_of_microbiology/volume_5_number_1_21/article/molasses _as_bifidus_promoter_o n_bifidobacteria_and_lactic_acid_bacteria_growin g_in_skim_milk.html (accessed February 13, 2009).

5. Kirschmann, J. D. Nutrition Almanac, 6th Ed.; McGraw Hill Books: New York, 2007; pp. 112-113.

6. Crellin, J. K.; Philpott, J.; Bass Tommie, A. L. Herbal Medicine Past and Present: A Reference Guide to Medicinal Plants; Duke University Press: Durham, NC, 1990; Vol. 2, pp. 405-406.

7. Preston, T. R.; Sansoucy, R.; Aarts, G. Molasses as Animal Feed: An Overview. FAO Expert Consultation on Sugarcane as Feed. FAO: Rome, 1986.

8. Lora, J.; Ravelo, G.; Minor, S., Preston, T. R.; Leng, R. A. Glucose Metabolism in Cattle on Molasses Based Diets: Studies on Molasses Toxicity. Trop. Anim. Prod. 1977, 3(1), 19-21.

9. Whitehouse, R. C.; Boullata, J.; McCauley, L. A. The Potential Toxicity of Artificial Sweeteners. AAOHN J. 2008, 56(6), 251-259.

10. Zygler, A.; Andrzej, W.; Namiesnik, J. Analytical Methodologies for Determination of Artificial Sweeteners in Foodstuffs. Trends Anal. Chem. 2009, 28(9), 1082-1102.

11. Arnold, D. L.; Krewki, D.; Munro, I. C. Saccharin: A Toxicological and Historical Perspective. Toxicology 1983, 27, 179-256.

12. Weihrauch, M. R.; Diehl, V. Artificial Sweeteners - Do They Bear a Carcinogenic Risk? Ann. Oncol. 2004, 15, 1460-1465.

13.Szucs, E. F.; Barrett, K. E.; Metcalfe, D. D. The Effects of Aspartame on Mast Cells and Basophils. Food Chem. Toxicol. 1986, 24(2), 171-174.

14. Grenby, T. H. Intense Sweeteners for the Food Industry: An Overview. Trends Food Sci. Technol. 1991, 2, 2-6.

15. Renwick, A. G. The Fate of Intense Sweeteners in the Body. Food Chem. 1985, 16, 281-301.

16. Olney, J. W.; Farber, N. B.; Spitznagel, E.; Robins, L. N. Increasing Brain Tumor Rates: Is There a Link to Aspartame? J. Neuropathol. Exp. Neurol. 1996, 55, 11151123 .

17. Pillai, R. M.; Watson, R. R. In vitro Immunotoxicology and Immunopharmacology: Studies on Drugs of Abuse. Toxicol. Lett. 1990, 53, 269283.

18.Ladics, G. S. Use of SRBC Antibody Responses for Immunotoxicity Testing. Methods 2007, 41, 9-19.

19. Nandi, D.; Sarin, A. Trends in Basic Immunology Research - 2001 and Beyond. Curr. Sci. 2001, 8o(5), 647-652. 
20. Burrell, R. Human Immune Toxicity. Mol. Aspects Med. 1993, 14(1), 1-81.

21.Vos, J.; Van Loveren, H.; Wester, P.; Vethaak, D. Toxic Effects of Environmental Chemicals on the Immune System. Trends Pharmacol. Sci. 1989, 1O(7), 289-292.

22. Colosio, C.; Birindelli, S.; Corsini, E.; Galli, C. L.; Maroni, M. Low-Level Exposure to Chemicals and Immune System. Toxicol. Appl. Pharmacol. 2005, 207, S320-328.

23. Langezaal, I.; Coecke, S.; Hartung, T. Whole Blood Cytokine as a Measure of Immunotoxicity. Toxicol. In Vitro 2001, 15, 313-318.

24. Hussain, R.; Kaleem, A.; Shahid, F.; Dojki, M.; Jamil, B.; Mehmood, H.; Dawood, G.; Dockrell, H. M. Cytokine Profiles using Whole-Blood Assays can Discriminate between Tuberculosis Patients and Healthy Endemic Controls in a BCG-vaccinated Population. J. Immunol. Meth. 2002, 264, 95-108.

25. Pool, E. J.; Robson, P. J.; Smith, C.; Van Wyk, J. H.; Myburgh, K. H. In vitro Interleukin-6 Release in Whole Blood Cultures in Samples taken at Rest from Triathletes and Professional Rugby Players. Eur. J. Appl. Physiol. 2002, 87, 233237.

26. Robles, T. F.; Glaser, R.; Janice, K. Out of Balance: A New Look at Chronic Stress, Depression and Immunity. Curr. Dir. Psychol. Sci. 2005, 2, 111-115.

27. Rahiman, F.; Pool, E. J. The Effects of Saccharum officinarium (Sugar Cane) Molasses on Cytokine Secretion by Human Blood Cultures. J. Immunoassay Immunochem. 2010, 31(2), 148-159.

28. Barak, V.; Birkenfeld, S.; Halperin, T.; Kalickman, I. The Effect of Herbal Remedies on the Production of Human Inflammatory and Anti-inflammatory Cytokines. Israel Med. Assoc. J. 2002, 4, 919-922.

29. Twigg, H. L. III. Humoral Immune Defense (Antibodies): Recent Advances. Proc. Amer. Thorac. Soc. 2005, 2, 417-421.

30. Langezaal, I.; Hoffman, S.; Hartung, T.; Coecke, S. Evaluation and Prevalidation of an Immunotoxicity Test based on Human Whole Blood Cytokine Release. Altern. Lab. Anim.2002, 30, 581-595. 